\title{
Perchlorination of Coronene Enhances its Propensity for Self-Assembly on Graphene
}

\author{
Simone Conti ${ }^{+}{ }^{[a]}$ Maria G. del Rosso ${ }_{,}^{[b]}$ Artur Ciesielski, ${ }^{[b]}$ Jürgen Weippert, ${ }^{[c]}$ Artur Böttcher, ${ }^{[c]}$ \\ Yuyoung Shin, ${ }^{[\mathrm{d}]}$ Georgian Melinte, ${ }^{[\mathrm{e}]}$ Ovidiu Ersen, ${ }^{[\mathrm{e}]}$ Cinzia Casiraghi, ${ }^{[\mathrm{dd}]}$ Xinliang Feng, ${ }^{[\mathrm{f}, \mathrm{g}]}$ \\ Klaus Müllen, ${ }^{[f]}$ Manfred M. Kappes, ${ }^{*[c]}$ Paolo Samori, ${ }^{*[b]}$ and Marco Cecchini ${ }^{*[a]}$
}

\begin{abstract}
Providing a quantitative understanding of the thermodynamics involved in molecular adsorption and self-assembly at a nanostructured carbon material is of fundamental importance and finds outstanding applications in the graphene era. Here, we study the effect of edge perchlorination of coronene, which is a prototypical polyaromatic hydrocarbon, on the binding affinity for the basal planes of graphite. First, by comparing the desorption barrier of hydrogenated versus perchlorinated coronene measured by temperature-programmed desorption, we quantify the enhancement of the strength of physisorption at the single-molecule level though chlorine substitution. Then, by a thermodynamic analysis of the corresponding monolayers based on force-field calculations and statistical mechanics, we show that perchlorination decreases the free energy of self-assembly, not only enthalpically (by enhancing the strength of surface binding), but also entropically (by decreasing the surface concentration). The functional advantage of a chemically modulated 2D self-assembly is demonstrated in the context of the molecule-assisted liquid-phase exfoliation of graphite into graphene.
\end{abstract}

Almost 100 years after the first derivation of the $C_{6} / R^{6}$ nature of dispersive interactions by London, ${ }^{[1]}$ a full understanding of these relatively weak intermolecular forces is still an outstanding challenge. ${ }^{[2]}$ At the same time, dispersive interactions are ubiquitous in nature and play a critical role in several contexts spanning from the structure of the DNA double helix ${ }^{[3]}$ to a gecko's walk. ${ }^{[4]}$ Harnessing dispersion interactions at the nanoscale through the chemical tailoring of molecular compo- nents would provide exquisite control over the interfacial forces and boost the development of bottom-up approaches in a number of technological applications and fields, including organic electronics ${ }^{[5]}$ and semiconductors, ${ }^{[6]}$ catalysis, $^{[7]}$ gas sensing ${ }^{[8]}$ and energy storage ${ }^{[9]}$ and conversion. ${ }^{[10]}$ In addition, as the strength of such noncovalent interactions is critically involved in supramolecular phenomena, such as molecular selfassembly at surfaces and interfaces, ${ }^{[11]}$ finely tuned recognition events are key to the nanofabrication of electronic circuits, ${ }^{[12]}$ sensing (of heavy metals), ${ }^{[13]}$ and the large-scale production of graphene. ${ }^{[14]}$

Milestone contributions towards the quantitative understanding of interfacial interactions at surfaces have primarily resulted from temperature-programmed desorption (TPD) studies. Early analyses of short linear alkanes $(n<12)$ have shown that the binding energy to the graphite surface increases roughly linearly with the number of carbon atoms, ${ }^{[15]}$ with each methylene group contributing approximately $2 \mathrm{kcal}$ $\mathrm{mol}^{-1}{ }^{[16]}$ At the same time, it has been found that the increased configurational entropy loss upon the physisorption of longer alkanes $(n>12)$ actually reduces the net binding energy per carbon atom and results in a nonlinear dependence of the desorption barrier on chain length. ${ }^{[17]}$ Corresponding analyses of a series of polycyclic aromatic hydrocarbons (PAHs) revealed that the more limited configurational freedom imposed by an extended electronic conjugation ensures a linear size dependence of the desorption barrier even for $n>30$, although the net binding energy per carbon atom decreases to approximately $1.2 \mathrm{kcal} \mathrm{mol}^{-1} .{ }^{[18]}$ Finally, TPD analyses of bromo-substituted $n$-alkanes and carboxylic acids on highly oriented pyro- [a] S. Conti, Dr. M. Cecchini

Laboratoire d'Ingénierie des Fonctions Moléculaires, ISIS \& icFRC Université de Strasbourg \& CNRS

8 allée Gaspard Monge, 67000 Strasbourg (France) E-mail:mcecchini@unistra.fr

[b] M. G. del Rosso, ${ }^{+}$Dr. A. Ciesielski, Prof. Dr. P. Samorì Nanochemistry Laboratory, ISIS \& iCFRC

Université de Strasbourg \& CNRS

8 allée Gaspard Monge, 67000 Strasbourg (France) E-mail: samori@unistra.fr

[c] J. Weippert, Dr. A. Böttcher, Prof. Dr. M. M. Kappes Institute of Physical Chemistry, Karlsruhe Institute of Technology (KIT) Fritz-Haber-Weg 2, 76131 Karlsruhe (Germany) E-mail:manfred.kappes@kit.edu

[d] Y. Shin, Dr. C. Casiraghi School of Chemistry, University of Manchester Oxford road, Manchester M13 9PL (UK) [e] G. Melinte, Prof. Dr. O. Ersen

Institut de Physique et Chimie des Matériaux de Strasbourg (IPCMS) UMR 7504, 23, rue du Loess, 67037 Cedex 08 Strasbourg (France)

[f] Prof. Dr. X. Feng, Prof. Dr. K. Müllen Max Plank Institute for Polymer Research Ackermannweg 10, 55128 Mainz (Germany)

[g] Prof. Dr. X. Feng Center for Advancing Electronics Dresden (CFAED) \& Department of Chemistry and Food Chemistry Technische Universitaet Dresden, 01062 Dresden (Germany)

${ }^{+}{ }^{+}$These authors contributed equally to this work

$\square$ Supporting Information and ORCID(s) from the author(s) for this article iD are available on the WWW under http://dx.doi.org/10.1002/ cphc.201501113. 
lytic graphite (HOPG) demonstrated that chemical functionalization, for example, monobromo substitution, may have a profound influence on the desorption barrier and result in a significant increase of the surface-binding affinity. ${ }^{[19]}$

Computational approaches based on molecular modeling have also been used to explore the energetics of dispersion interactions at nanostructured carbon materials. ${ }^{[20]}$ Remarkably, dispersion-corrected density functional theory (DFT $)^{[21]}$ and, more recently, also semiempirical quantum mechanics ${ }^{[22]}$ and force-field calculations, ${ }^{[23]}$ were shown to predict desorption energies for a series of small-molecule adsorbates on graphite and were in quantitative agreement with TPD results. More challenging, however, is the first-principle interpretation of molecular self-assembly at surfaces, which requires an accurate description of dispersive interactions in molecular systems involving thousands of atoms. Towards this goal, two main computational strategies have been put forward. On the one hand, high level of theory calculations based on dispersion-corrected DFT have been used to explore the energetics of $2 \mathrm{D}$ self-assembly under rather strong approximations, which were introduced to reduce the computational burden, and include ignoring the presence of the substrate, ${ }^{[24]}$ use of periodic boundaries by imposing the symmetry of the self-assembled monolayer (SAM) to a not necessarily commensurable substrate, ${ }_{1}^{[25]}$ or neglecting entropy changes. On the other hand, computational approaches based on force-field calculations and statistical mechanics were shown by $\mathrm{us}^{[26]}$ and others ${ }^{[27]}$ to provide a measure of the thermodynamic stability of the SAM, in some cases in quantitative agreement with experiments. ${ }^{[28]}$ Although the quality of these predictions is inherently dependent on the parameterization of the underlying force field, these methods are naturally open to very large system sizes and enable a firstprinciple interpretation of the equilibrium structure of the SAM, including entropic effects.

In this Communication, we report on the interesting observation that coronene self-assembly on graphite/graphene is aided by its chemical functionalization through edge perchlorination. Motivated by computational analysis of the strength of the binding to graphite of hydrogenated $\left(\mathrm{C}_{24} \mathrm{H}_{12}\right)$ versus perchlorinated $\left(\mathrm{C}_{24} \mathrm{Cl}_{12}\right)$ coronene (see Scheme 1), we compared their desorption barriers by using TPD and analyzed the effect of perchlorination on supramolecular organization on graphene. We find that the introduction of highly-polarizable chlorine substituents at the molecular periphery of coronene enhances its propensity to 2D self-assembly, not only by increasing the strength of surface binding, but also by reducing the surface concentration, that is, the number of molecules per unit of surface area, which is an entropic effect. The chemically enhanced molecule-surface interaction improves the ability of coronene to act as a dispersion-stabilizing agent to aids the exfoliation of graphite in liquid media. Our analysis demonstrates that an appropriate combination of modeling and statistical mechanics may provide unprecedented insights into the fundamental nature of molecular self-assembly at surfaces and interfaces.

The strength of molecular binding to graphite was first examined through modeling. Following recent work by $u s^{[23]}$ the desorption energy of $\mathrm{C}_{24} \mathrm{H}_{12}$ and $\mathrm{C}_{24} \mathrm{Cl}_{12}$ were computed by subtracting the energy of both the adsorbate and the substrate in isolation from that of the complex upon geometry optimization. The calculations were carried out by using an atomistic representation of the substrate and modeling physisorption using both the CGenFF classical force field ${ }^{[29]}$ and the dispersion-corrected semiempirical method $\mathrm{PM6}-\mathrm{DH}+;^{[30]}$ these methods were recently shown to predict desorption energies from graphite in quantitative agreement with TPD data. ${ }^{[23]}$ The computational results are given in Table 1. At this stage, higher level of theory calculations were not considered, because the size of substrate/adsorbate complex is prohibitive for most

\begin{tabular}{|c|c|c|c|}
\hline Measured quantity & $\mathrm{C}_{24} \mathrm{H}_{12}$ & $\mathrm{C}_{24} \mathrm{Cl}_{12}$ & $\Delta$ \\
\hline$E_{\text {des }}(\mathrm{PM6}-\mathrm{DH}+)\left[\mathrm{kcal} \mathrm{mol}^{-1}\right]$ & 41.4 & 69.4 & 28.0 \\
\hline$E_{\text {des }}\left(\right.$ CGenFF) $\left[\mathrm{kcal} \mathrm{mol}^{-1}\right]$ & 36.1 & 45.2 & 9.1 \\
\hline$E_{\text {des }}(\mathrm{TPD})\left[\mathrm{kcal} \mathrm{mol}^{-1}\right]$ & 36.9 & 41.5 & 4.6 \\
\hline$v(\mathrm{TPD})\left[\mathrm{s}^{-1}\right]$ & $10^{16}$ & $2.8 \times 10^{16}$ & - \\
\hline$A_{u c}(C G e n F F)\left[\AA^{2}\right]$ & 110.88 & 143.81 & $30 \%$ \\
\hline$\gamma\left(\right.$ CGenFF) $\left[\mathrm{kcal} \mathrm{mol}^{-1} \mathrm{~nm}^{-2}\right]$ & -19.4 & -22.9 & -3.5 \\
\hline graphene concentration $^{[a]}\left[\mu \mathrm{g} \mathrm{mL}^{-1}\right]$ & $95 \pm 6$ & $128 \pm 2$ & $+33 \pm 6$ \\
\hline graphene concentration $^{[\mathrm{b}]}\left[\mu \mathrm{g} \mathrm{mL}^{-1}\right]$ & $114 \pm 20$ & $171 \pm 1$ & $+57 \pm 20$ \\
\hline SLG (TEM) [\%] & 9 & 13 & +4 \\
\hline FLG (TEM) [\%] & 60 & 74 & +14 \\
\hline
\end{tabular}

The concentration of dispersed graphene was quantified by [a] filtering through a polytetrafluoroethylene (PTFE) membrane (pore size of $0.1 \mu \mathrm{m}$ ) and weighing the filtered mass on a microbalance, or $[b]$ measuring the UV/Vis adsorption at $\lambda=660 \mathrm{~nm}$ by using the absorption coefficient of $2460 \mathrm{~mL} \mathrm{mg}^{-1} \mathrm{~m}^{-1} \cdot{ }^{[14 a]}$<smiles></smiles><smiles></smiles>

Scheme 1. Structures of coronene $\left(\mathrm{C}_{24} \mathrm{H}_{12}\right)$ and perchlorocoronene $\left(\mathrm{C}_{24} \mathrm{Cl}_{12}\right)$. ab initio methods and the accuracy of DFT in surface-adsorption problems may also be model dependent, even when corrections for dispersion interactions are introduced. ${ }^{[21 a]}$ Both models (i.e. CGenFF and PM6-DH+) consistently predicted a significant increase of the desorption barrier upon perchlorination, although the enhancement found by $\mathrm{PM6}-\mathrm{DH}+$ was three times larger. Interestingly, a DFT analysis $(\omega B 97 X-D)^{[31]}$ of the strain associated with planarization of perchlorocoronene in isolation validated the force-field results and showed that 
the much stronger adsorption predicted by $\mathrm{PM6}-\mathrm{DH}+$ originates from a significant underestimation of the intramolecular strain on physisorption (see the Supporting Information). Therefore, based on the force-field results, we predict that perchlorination of coronene introduces a $25 \%$ enhancement of the binding energy to graphite. As the isotropic distribution of the chlorine substituents cannot introduce a net molecular dipole, the observed increase of the binding affinity is solely attributed to the large polarizability of the chlorine substituents, which results in a two-fold increase of the isotropic polarizability of the molecule, as evaluated by the finite-field approach at the semiempirical level ${ }^{[32]}$ (see the Supporting Information). Finally, structural analysis of the optimized geometries on the surface revealed a striking difference in the adsorption complexes formed by $\mathrm{C}_{24} \mathrm{H}_{12}$ versus $\mathrm{C}_{24} \mathrm{Cl}_{12}$, with the former lying flat on the surface and the latter adopting a configuration with pairs of peri-related chlorine atoms flipped up and down relative to the plane of the molecule (Figure 1). This result is consistent with the $\mathrm{X}$-ray structure of perchlorocoronene $\mathrm{e}^{[33]}$ and several expanded perchlorinated PAHs. ${ }^{[34]}$ Interestingly, the

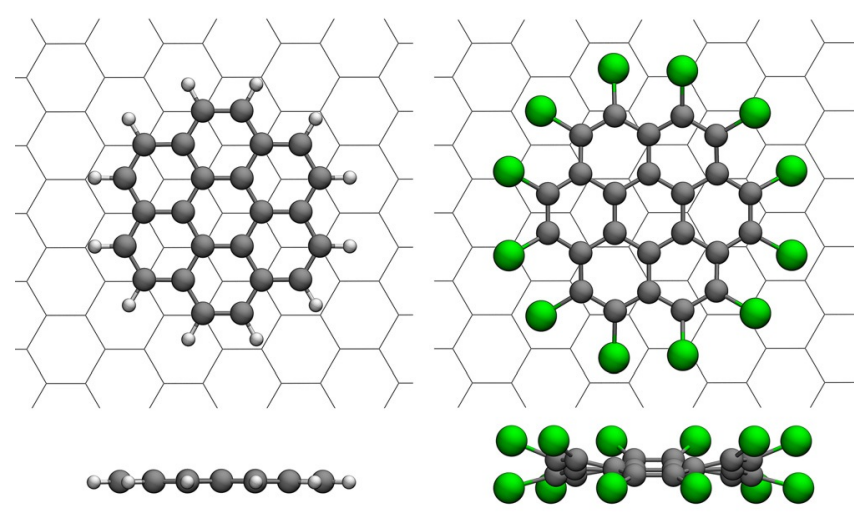

Figure 1. Top and side views of modeled coronene (left) and perchlorocoronene (right) on graphene, optimized by CGenFF. Grey, white, and green spheres represent carbon, hydrogen, and chlorine atoms, respectively. The adsorbed configuration of $\mathrm{C}_{24} \mathrm{Cl}_{12}$ shows a significant distortion from the planar geometry.

nonplanar configuration of $\mathrm{C}_{24} \mathrm{Cl}_{12}$ in the bound state indicates that edge perchlorination gives rise to a competition between the (enhanced) strength of binding to the substrate and the internal strain of the adsorbate, which distorts to accommodate the sterically more demanding substituents. Consistently, reoptimization of $\mathrm{C}_{24} \mathrm{Cl}_{12}$ in isolation revealed an unfavorable strain of $2.1 \mathrm{kcal} \mathrm{mol}^{-1}$ in the molecular geometry adopted by the surface-bound complex (see the Supporting Information).

The adsorption-energy enhancement predicted by the computations was tested experimentally by measuring the binding energy of $\mathrm{C}_{24} \mathrm{H}_{12}$ and $\mathrm{C}_{24} \mathrm{Cl}_{12}$ to HOPG by using TPD. ${ }^{[35]}$ For this purpose, solid powders of $\mathrm{C}_{24} \mathrm{H}_{12}$ and $\mathrm{C}_{24} \mathrm{Cl}_{12}$ were prepared and used to grow submonolayer films on clean HOPG under ultrahigh-vacuum (UHV) conditions by: 1) sublimation from a Knudsen cell after extended degassing or 2) low-energy ion- beam deposition of the parent cations by electron-impact ionization and mass-to-charge selection (LECBD; see the Supporting Information) $)^{[36]}$. TPD spectra, that is, desorption rate versus sample temperature measurements, were recorded at well-defined initial coverage $\left(\Theta_{i}<1\right)$ and at a constant heating rate $(\beta)$ of $2.5 \mathrm{~K} \mathrm{~s}^{-1}$. Figure 2 shows that although the desorption rate of both $\mathrm{C}_{24} \mathrm{H}_{12}$ and $\mathrm{C}_{24} \mathrm{Cl}_{12}$ is strongly dependent on the initial coverage, the value of the temperature at the maximum

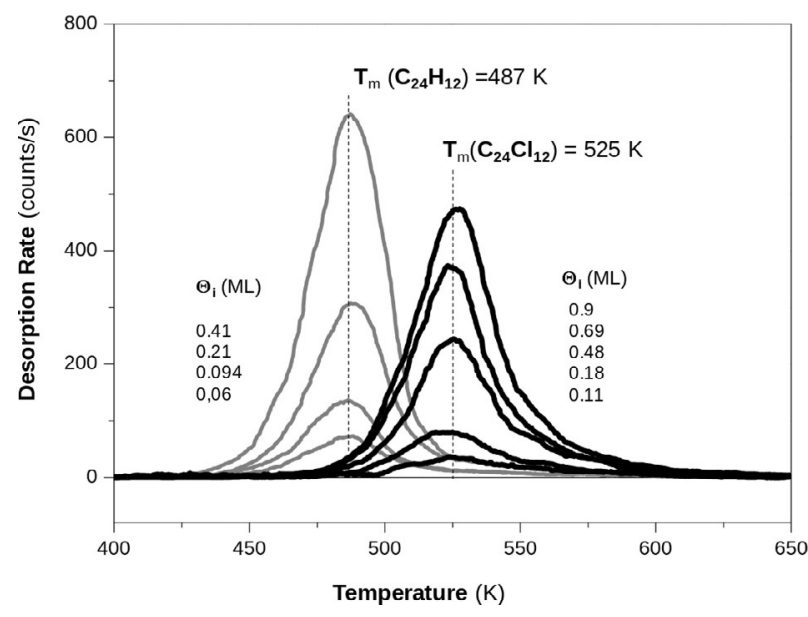

Figure 2. TPD spectra obtained for several $\mathrm{C}_{24} \mathrm{H}_{12}$ and $\mathrm{C}_{24} \mathrm{Cl}_{12}$ adlayers at different initial coverage $\left(\Theta_{i}\right.$; as indicated in the figure). All desorption profiles were obtained at a constant heating rate of $2.5 \mathrm{~K} \mathrm{~s}^{-1}$. The shift in the desorption temperature $T_{\mathrm{m}}$ indicates that perchlorination results in an increase in the binding energy.

$\left(T_{\mathrm{m}}\right)$ is not, thus indicating that the lateral interaction between adjacent molecules is negligible in comparison with adsorbate/ substrate bonding. Moreover, the spectra evidence first-order desorption kinetics, which allows for an estimation of the activation energy for desorption by using the Redhead equation, $E_{\text {des }}=k T_{\mathrm{m}}\left[\ln \left(v T_{\mathrm{m}} / \beta\right)-3.46\right]$, where kis the Boltzmann constant ${ }^{[36]}$ If the same value of the preexponential factor, $v$, is assumed for both molecules, the observation that $T_{\mathrm{m}}\left(\mathrm{C}_{24} \mathrm{H}_{12}\right)<T_{\mathrm{m}}\left(\mathrm{C}_{24} \mathrm{Cl}_{12}\right)$, as evidenced by Figure 2, already implies that $E_{\text {des }}\left(C_{24} H_{12}\right)<E_{\text {des }}\left(C_{24} C_{12}\right)$. However, to quantify the surface-binding enhancement resulting from the edge perchlorination of coronene, molecule specific preexponential factors were determined from a series of measurements of $T_{\mathrm{m}}$ over a wide range of heating rates, that is, $0.5-17 \mathrm{~K} \mathrm{~s}^{-1}$, for both adlayers. By plotting $\ln \left(\beta / T_{\mathrm{m}}{ }^{2}\right)$ versus $\left(1 / k T_{\mathrm{m}}\right)$, a quantitative estimate of surface-binding enhancement through perchlorination was obtained; see Table 1. The results show that the preexponential factor $(v)$ for $\mathrm{C}_{24} \mathrm{Cl}_{12}$ is nearly three times larger $\left(v \approx 2.8 \times 10^{16} \mathrm{~s}^{-1}\right)$, which in turns corresponds to a desorption energy that is $4.6 \mathrm{kcal} \mathrm{mol}^{-1}$ higher than that of $\mathrm{C}_{24} \mathrm{H}_{12}$, which is consistent with the computational findings.

The supramolecular advantages of edge perchlorination of coronene were analyzed in the context of its self-assembly on graphite/graphene surfaces. Model architectures with atomic resolution were built for both $\mathrm{C}_{24} \mathrm{Cl}_{12}$ and $\mathrm{C}_{24} \mathrm{H}_{12}$ (Figure 3) based on the 2D hexagonal lattice of coronene self-assembly 


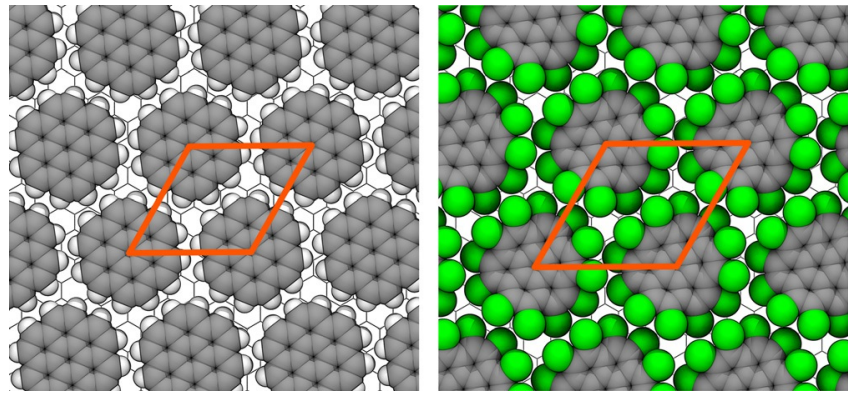

Figure 3. Modeled SAMs of coronene (left) and perchlorocoronene (right) on graphene. Both architectures adopt hexagonal lattices with cell sizes of 11.32 and $12.89 \AA$ and unit-cell areas of 110.88 and $143.81 \AA^{2}$, respectively.

determined by scanning tunneling microscopy (STM) in UHV $\mathrm{V}^{[37]}$ (unit cell parameters: $a=b=11.28 \AA, \alpha=60^{\circ}$, area $=110 \AA^{2}$ ). Upon geometry optimization, the unit-cell parameters of the SAM formed by $\mathrm{C}_{24} \mathrm{H}_{12}$ on single-layer graphene (SLG) were in quantitative agreement with those observed with STM. ${ }^{[37]}$ By contrast, the SAM produced by $\mathrm{C}_{24} \mathrm{Cl}_{12}$, for which no STM analysis was available, showed an increase of the unit-cell area by $30 \%$, due to the bulky chlorine substituents at the molecular periphery (see Table 1 for the $A_{\mathrm{uc}}$ values). The thermodynamic stability of the modeled architectures was then assessed by comparing their surface free-energy excess $(\gamma)$, which corresponds to the reversible work per unit of area to cover the substrate through the noncovalent association of freely-diffusing monomers into a 2D crystalline architecture (see the Supporting Information). Following our previous work, ${ }^{[26]}$ the energy gain on forming the SAM, which includes contributions from the (vertical) molecule-substrate interactions, the (lateral) molecule-molecule interactions, and the intramolecular strain associated with molecular planarization on the surface, was quantified by force-field (CGenFF) calculations, which uniquely allow the exploration of the energetics of (supra)molecular systems made up of several thousands of atoms (see the Supporting Information). In addition, the entropy cost of confining monomers to the 2D lattice was evaluated by equilibrium statistical mechanics within the limits of the ideal gas approximation (see the Supporting Information). The results of the analysis indicate that at $300 \mathrm{~K}$ and $1 \mathrm{M}$ concentration of the monomers, $\gamma$ is $3.5 \mathrm{kcal} \mathrm{mol}^{-1} \mathrm{~nm}^{-2}$ more favorable for $\mathrm{C}_{24} \mathrm{Cl}_{12}$, thus indicating that the perchlorinated SAM is thermodynamically most stable. Surprisingly, the decomposition of $\gamma$ into energy and entropy contributions indicates that both of them favor the self-assembly of $\mathrm{C}_{24} \mathrm{Cl}_{12}$, accounting for 1.4 and $2.1 \mathrm{kcal}$ $\mathrm{mol}^{-1} \mathrm{~nm}^{-2}$ of the surface free-energy excess difference, respectively (see the Supporting Information). This suggests that the formation of perchlorinated SAM is energetically more favored because of the stronger interaction with the substrate. This finding is consistent with the TPD analysis, as well as stronger cohesion forces within the monolayer, which arise from chlorine-chlorine intermolecular dispersive interactions. However, and perhaps surprisingly, it also indicates that the presence of sterically more demanding substituents (chlorine) at the molecular periphery produces a significant expansion of the unit-cell area of the SAM, thus effectively reducing the number of monomers required to cover a given surface area. Interestingly, the latter results in a decreased (translation and rotational) entropy loss upon self-assembly.

Finally, we demonstrate a connection between the computational and experimental results above with the ability of $\mathrm{C}_{24} \mathrm{Cl}_{12}$ versus that of $\mathrm{C}_{24} \mathrm{H}_{12}$ to act as dispersion-stabilizing agents in the liquid-phase exfoliation (LPE) of graphite, which stands out as a versatile and up-scalable approach to the production of high-quality graphene. ${ }^{[14 a]}$ Graphite LPE typically involves: 1) the dispersion of graphite in solvents with a surface tension of $30-40 \mathrm{~mJ} \mathrm{~m}^{-2}, 2$ ) the exfoliation of the 2D-layered material assisted by sonication, and 3) the purification of the exfoliated product. ${ }^{[14 b]}$ Recently, it was shown by us that the yield of exfoliation in organic media can be significantly improved by the addition of dispersion-stabilizing agents such as linear alkanes $^{[38]}$ or fatty acids, ${ }^{[26 a]}$ which prevent restacking by self-assembly on the exfoliated sheets. For this purpose, graphite powder dispersions prepared in ortho-dichlorobenzene (o$D C B)^{[39]}$ in the presence of $\mathrm{C}_{24} \mathrm{H}_{12}$ or $\mathrm{C}_{24} \mathrm{Cl}_{12}$ were sonicated for $6 \mathrm{~h}$ at $40^{\circ} \mathrm{C}$, decanted, and centrifuged until homogeneous solutions were obtained (see the Supporting Information). The concentration of graphene in the dispersion was then quantified (Table 1). The results indicate a considerable improvement of the exfoliation yield (from 34 to $50 \%$ ) upon exchanging coronene with perchlorocoronene (see the Supporting Information for $Y_{\mathrm{w}}$ values, where $Y_{\mathrm{w}}$ is the yield of exfoliation defined as the ratio between the weight of dispersed graphitic materials and that of the starting graphite flakes). Furthermore, the exfoliated material was characterized by counting the number of graphitic planes at the folded edges of the exfoliated sheets by visual inspection of high-resolution transmission electron microscopy (HR-TEM) micrographs. The result of the analysis of hundreds of flakes (Figure 4) shows that the use of $\mathrm{C}_{24} \mathrm{Cl}_{12}$ during LPE increases the amount of SLG and few-layer graphene (FLG) sheets by approximately 4 and $14 \%$, respectively, relative to dispersions prepared in the presence of $\mathrm{C}_{24} \mathrm{H}_{12}$. Similar results were obtained by micro Raman spectroscopy, where
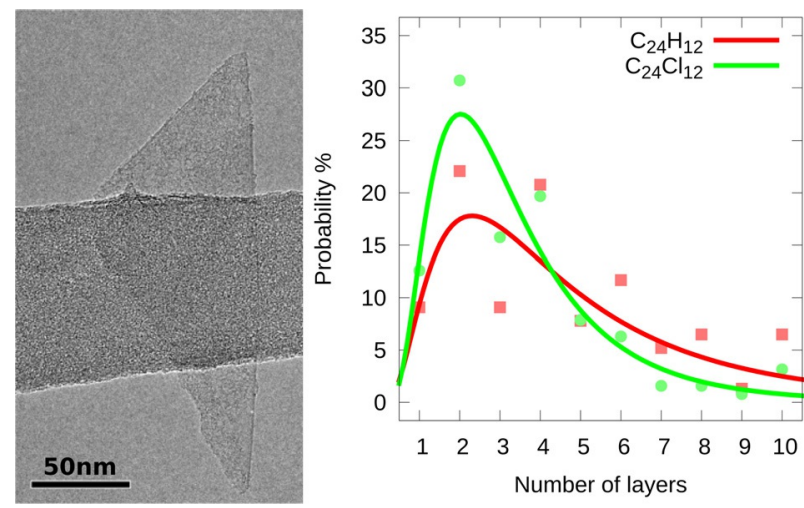

Figure 4. Left: HR-TEM image of an exfoliated bilayer graphene. Right: The statistical analysis of exfoliated graphene in the presence of $\mathrm{C}_{24} \mathrm{H}_{12}$ (red) or $\mathrm{C}_{24} \mathrm{Cl}_{12}$ (green). Data were collected by visual inspection of hundreds of HRTEM micrographs. 
both the relative intensity of the $D$ versus $G$ peaks and the determination coefficient by fitting the $2 \mathrm{D}$ peak to a Lorentzian, $R^{2}(2 D)$, showed a notable increase of SLG in the presence of $\mathrm{C}_{24} \mathrm{Cl}_{12}$ (see the Supporting Information). Consistently, both the HR-TEM and Raman results support the conclusion that the use of $\mathrm{C}_{24} \mathrm{Cl}_{12}$ improves the quality of the exfoliation by increasing the population of SLG and FLG sheets. Finally, by combining the experimental determinations of exfoliated graphene (Table 1) with the results of the HR-TEM analysis, one finds that the use of $\mathrm{C}_{24} \mathrm{Cl}_{12}$ doubles the production of highquality graphene (SLG) relative to $\mathrm{C}_{24} \mathrm{H}_{12}$.

By taking advantage of the atomistic description of interactions provided by the modeling studies, both the TPD and LPE experimental findings above could be interpreted at the molecular level. First, the TPD analysis of $\mathrm{C}_{24} \mathrm{Cl}_{12}$ versus $\mathrm{C}_{24} \mathrm{H}_{12}$ showed that coronene perchlorination results in a $12.5 \%$ increase of the desorption energy from graphite. Our calculations indicate that the surface-affinity enhancement of $\mathrm{C}_{24} \mathrm{Cl}_{12}$ mainly arises from the presence of highly polarizable groups, which strengthen the binding to graphite through dispersive interactions. Also, they suggest that the surface-binding enhancement through edge perchlorination is somewhat suboptimal, as the introduction of bulky substituents at the molecular periphery results in a highly nonplanar configuration, the partial flattening of which on the surface introduces intramolecular strain that is equivalent to $23 \%$ of the total increase in binding energy. By using the same setup, the hypothetical, partially chlorinated $1,2,5,6,9,10$-hexachlorocoronene, which is flat both on the surface and in isolation, is predicted to bind stronger to graphite by $3 \mathrm{kcal} \mathrm{mol}^{-1}$ (see the Supporting Information). Therefore, regioselective chlorine substitution on PAHs, which still represents a major synthetic challenge, may further enhance the surface-binding affinity for graphite/ graphene.

Second, the LPE results indicate that both the quantity and the quality of the exfoliated material can be influenced by the chemical modulation of the strength of the adsorbate/substrate interactions. Our thermodynamic analysis based on atomistic models of the SAM and equilibrium statistical mechanics predicts that the introduction of chlorine substituents favors $2 \mathrm{D}$ self-assembly not only by strengthening the interactions with the substrate and within the monolayer, but also by reducing the surface concentration, that is, the number of molecules per unit of surface area. In fact, the presence of sterically demanding groups at the periphery of the molecule produces a significant expansion of the unit cell of the SAM, which results in decreased molecular density at the surface, and therefore, a lower (translational and rotational) entropy loss upon self-assembly. It follows that perchlorination of coronene improves the exfoliation ability by stabilizing the formation of the SAM not only enthalpically (strengthened interactions), but also entropically (decreased surface concentration). As a bonus, perchlorination enhances the solubility, and thus, the processability of $\mathrm{PAHs}^{\left[{ }^{[34]}\right.}$ which advocates the use of even larger (perchlorinated) PAHs in LPE as entropically-stabilized graphite exfoliators.
In conclusion, the results presented herein demonstrate that the chemical functionalization of PAHs through edge perchlorination enhances physisorption at nanostructured carbon materials by increasing the overall thermodynamic stability of the SAM. By disentangling the enthalpic versus entropic contributions to such stabilization, which was made possible by an original combination of atomistic modeling and statistical mechanics, we shed new light onto the fundamental nature of molecular self-assembly at surfaces, which will be useful for the design of improved surface-binding agents. The modulation of adsorbate/substrate interactions by chemical tailoring, which was exemplified by the perchloro substitution of coronene, emerges as a promising approach to shorten and simplify the large-scale production of high-quality graphene in liquid media for technological applications and boost the development of bottom-up molecular strategies in sensing, catalysis, and (opto)electronics.

\section{Acknowledgements}

This work was supported by the European Commission through the Graphene Flagship (GA-604391), the Agence Nationale de la Recherche through the LabEx project Chemistry of Complex Systems (ANR-10-LABX-0026 CSC), the International Center for Frontier Research in Chemistry (icFRC) and the German Science Foundation (DFG KA 972/9-1) and granted access to the HPC resources of CCRT under the allocation 2014-[076644] made by Grand Equipment National de Calcul Intensif (GENCI). S.C. received financial support from IdEx Investissements d'Avenir (Grant No. 16141). C.C. and Y.S. acknowledge support by the Engineering and Physical Sciences Research Council (EPSRC) in the framework of the project Graphene-Based Membranes (EP/K016946/1).

Keywords: graphene • interfaces - polycyclic aromatic hydrocarbons · self-assembly $\cdot$ statistical thermodynamics

[1] F. London, Z. Phys. 1930, 63, 245.

[2] S. Grimme, Angew. Chem. Int. Ed. 2008, 47, 3430; Angew. Chem. 2008, 120, 3478.

[3] J. Černý, M. Kabeláč, P. Hobza, J. Am. Chem. Soc. 2008, 130, 16055.

[4] K. Autumn, M. Sitti, Y. A. Liang, A. M. Peattie, W. R. Hansen, S. Sponberg, T. W. Kenny, R. Fearing, J. N. Israelachvili, R. J. Full, Proc. Natl. Acad. Sci. USA 2002, 99, 12252.

[5] a) R. M. Westervelt, Science 2008, 320, 324; b) H. Huang, S. Chen, X. Gao, W. Chen, A. T. S. Wee, ACS Nano 2009, 3, 3431; c) J.D. Emery, Q. H. Wang, M. Zarrouati, P. Fenter, M. C. Hersam, M. J. Bedzyk, Surf Sci 2011, 605, 1685.

[6] a) P. Lauffer, K. V. Emtsev, R. Graupner, T. Seyller, L. Ley, Phys Status Solidi B 2008, 245, 2064; b) Q. H. Wang, M. C. Hersam, Nat. Chem. 2009, 1, 206.

[7] G. M. Scheuermann, L. Rumi, P. Steurer, W. Bannwarth, R. Mülhaupt, J. Am. Chem. Soc. 2009, 131, 8262.

[8] F. Schedin, A. K. Geim, S. V. Morozov, E. W. Hill, P. Blake, M. I. Katsnelson, K. S. Novoselov, Nat. Mater. 2007, 6, 652.

[9] M. Pumera, Energy Environ. Sci. 2011, 4, 668.

[10] P. V. Kamat, J. Phys. Chem. Lett. 2011, 2, 242.

[11] a) C. A. Palma, M. Cecchini, P. Samorì, Chem. Soc. Rev. 2012, 41, 3713; b) J. M. MacLeod, F. Rosei, Small 2014, 10, 1038.

[12] J. Puigmartí-Luis, A. Minoia, H. Uji-i, C. Rovira, J. Cornil, S. De Feyter, R. Lazzaroni, D. B. Amabilino, J. Am. Chem. Soc. 2006, 128, 12602. 
[13] T. Zhang, Z. Cheng, Y. Wang, Z. Li, C. Wang, Y. Li, Y. Fang, Nano Lett. 2010, 10, 4738.

[14] a) Y. Hernandez, V. Nicolosi, M. Lotya, F. M. Blighe, Z. Sun, S. De, I. T. Mc Govern, B. Holland, M. Byrne, Y. K. Gun'Ko, J. J. Boland, P. Niraj, G. Duesberg, S. Krishnamurthy, R. Goodhue, J. Hutchison, V. Scardaci, A. C. Ferrari, J. N. Coleman, Nat. Nanotechnol. 2008, 3, 563; b) A. Ciesielski, P. Samorì, Chem. Soc. Rev. 2014, 43, 381.

[15] K. R. Paserba, A. J. Gellman, Phys. Rev. Lett. 2001, 86, 4338.

[16] S. L. Tait, Z. Dohnalek, C. T. Campbell, B. D. Kay, J. Chem. Phys. 2006, 125, 234308.

[17] K. R. Paserba, A. J. Gellman, J. Chem. Phys. 2001, 115, 6737.

[18] R. Zacharia, H. Ulbricht, T. Hertel, Phys. Rev. B 2004, 69, 155406.

[19] T. Müller, G. W. Flynn, A. T. Mathauser, A. V. Teplyakov, Langmuir 2003 19, 2812.

[20] M. Pykal, P. Jurecka, F. Karlicky, M. Otyepka, Phys. Chem. Chem. Phys., DOI: $10.1039 /$ C5CP03599F.

[21] a) J. Björk, F. Hanke, C.-A. Palma, P. Samorì, M. Cecchini, M. Persson, J. Phys. Chem. Lett. 2010, 1, 3407; b) R. Erni, M. D. Rossell, M.-T. Nguyen, S Blankenburg, D. Passerone, P. Hartel, N. Alem, K. Erickson, W. Gannett, A. Zettl, Phys. Rev. B 2010, 82, 165443.

[22] E. G. Gordeev, M. V. Polynski, V. P. Ananikov, Phys. Chem. Chem. Phys. 2013, 15, 18815.

[23] S. Conti, M. Cecchini, J. Phys. Chem. C 2015, 119, 1867.

[24] a) W. Mamdouh, R. E. A. Kelly, M. D. Dong, L. N. Kantorovich, F. Besenbacher, J. Am. Chem. Soc. 2008, 130, 695; b) I. Bald, Y. G. Wang, M. D. Dong, C. B. Rosen, J. B. Ravnsbaek, G. L. Zhuang, K. V. Gothelf, J. G. Wang, F. Besenbacher, Small 2011, 7, 939.

[25] M. El Garah, A. Dianat, A. Cadeddu, R. Gutierrez, M. Cecchini, T. R. Cook, A. Ciesielski, P. J. Stang, G. Cuniberti, P. Samorì, Small, DOI: 10.1002/ smll.201502957.

[26] a) S. Haar, A. Ciesielski, J. Clough, H. Yang, R. Mazzaro, F. Richard, S. Conti, N. Merstorf, M. Cecchini, V. Morandi, C. Casiraghi, P. Samorì, Small 2015, 11, 1691; b) S. Bonacchi, M. El Garah, A. Ciesielski, M. Herder, S Conti, M. Cecchini, S. Hecht, P. Samorì, Angew. Chem. Int. Ed. 2015, 54 4865; Angew. Chem. 2015, 127, 4947.
[27] a) J. F. Dienstmaier, K. Mahata, H. Walch, W. M. Heckl, M. Schmittel, M. Lackinger, Langmuir 2010, 26, 10708; b) C. Meier, M. Roos, D. Kunzel, A. Breitruck, H. E. Hoster, K. Landfester, A. Gross, R. J. Behm, U. Ziener, J Phys Chem. C 2010, 114, 1268.

[28] W. T. Song, N. Martsinovich, W. M. Heckl, M. Lackinger, J. Am. Chem. Soc 2013, 135, 14854

[29] K. Vanommeslaeghe, E. Hatcher, C. Acharya, S. Kundu, S. Zhong, J. Shim, E. Darian, O. Guvench, P. Lopes, I. Vorobyov, A. D. Mackerell, J. Comp. Chem. 2010, 31, 671.

[30] M. Korth, J. Chem. Theory Comput. 2010, 6, 3808.

[31] J. D. Chai, M. Head-Gordon, Phys. Chem. Chem. Phys. 2008, 10, 6615.

[32] H. A. Kurtz, J. J. P. Stewart, K. M. Dieter, J. Comput. Chem. 1990, 11, 82.

[33] T. Baird, J. H. Gall, D. D. Macnicol, P. R. Mallinson, C. R. Michie, J. Chem. Soc. Chem. Commun. 1988, 1471.

[34] Y. Z. Tan, B. Yang, K. Parvez, A. Narita, S. Osella, D. Beljonne, X. Feng, K. Müllen, Nat. Commun. 2013, 4, 2646.

[35] A. M. de Jong, J. W. Niemantsverdriet, Surf. Sci. 1990, 233, 355.

[36] A. Böttcher, P. Weis, A. Bihlmeier, M. M. Kappes, Phys. Chem. Chem. Phys. 2004, 6, 5213.

[37] J. D. Thrower, E. E. Friis, A. L. Skov, L. Nilsson, M. Andersen, L. Ferrighi, B. Jorgensen, S. Baouche, R. Balog, B. Hammer, L. Hornekaer, J. Phys. Chem. C 2013, 117, 13520.

[38] A. Ciesielski, S. Haar, M. El Gemayel, H. Yang, J. Clough, G. Melinte, M. Gobbi, E. Orgiu, M. V. Nardi, G. Ligorio, V. Palermo, N. Koch, O. Ersen, C. Casiraghi, P. Samorì, Angew. Chem. Int. Ed. 2014, 53, 10355; Angew. Chem. 2014, 126, 10523.

[39] C. E. Hamilton, J. R. Lomeda, Z. Sun, J. M. Tour, A. R. Barron, Nano Lett. 2009, 9, 3460.

Manuscript received: December 3, 2015

Accepted Article published: December 14, 2015

Final Article published: December 23, 2015 